\title{
Configurable Phase/Amplitude Modulator Circuit based on Silicon Plasma Dispersion
}

\author{
Hong Deng, Wim Bogaerts \\ 1.Photonics Research Group, Ghent University - imec, Department of Information Technology, \\ Technologiepark-Zwijnaarde 126, 9052 Gent, BELGIUM \\ 2.Center for Nano and Biophotonics (NB-Photonics), Ghent University, Ghent, BELGIUM \\ Email: hong.deng@ugent.be
}

\begin{abstract}
A configurable modulator is demonstrated, implemented as a silicon Mach-Zehnder interferometer with a plasma dispersion modulator and tunable couplers. The configurable modulator can work as a phase modulator or intensity modulator with optimized modulation response.
\end{abstract}

\section{INTRODUCTION}

Photonic integrated circuits are increasingly being used in data communication systems and microwave photonic systems [1]. In these applications, electro-optic modulators are important. In most silicon photonics technologies, the standard phase modulator implementation is based on the plasma dispersion effect in a $P(I) N$ junction embedded into a waveguide [2]. This phase modulation is easily converted into intensity modulation by using a Mach-Zehnder interferometer (MZI) or microring resonator. As they are compatible with CMOS technology, plasma dispersion modulators have become the most popular solution and are provided in the process design kits by most foundries. However, the plasma dispersion effect does not induce high-quality phase modulation, introducing spurious intensity modulation and a nonlinear response.

Also, with the rising popularity of coherent communication formats and microwave photonics, the requirements of the modulators get more diverse: Some applications need amplitude modulation while others need phase modulation, and this with different dynamic range and linearity. In this paper, we demonstrate a configurable modulator circuit based on the plasma dispersion effect, which can be tuned to work as either an intensity modulator or a phase modulator[3]. The configurable modulator is implemented by embedding a standard PN junction modulator into a tunable MZI which consists of two TCs and a phase shifter. By tuning the TCs and the phase shifter, the phase and amplitude response of the modulator circuit can be reconfigured, adjusting the phase modulation range, intensity modulation range, insertion loss and modulation nonlinearity. Experimental results show that it can work as a phase modulator with a phase change of $0.3 \pi$, or as an intensity modulator with a $13 d B$ extinction ratio, within a reversed DC bias voltage from 0 to $10 \mathrm{~V}$, while it can also provide a pure phase modulation with a spurious intensity modulation of $0.06 \mathrm{~dB}$ within the same voltage range.

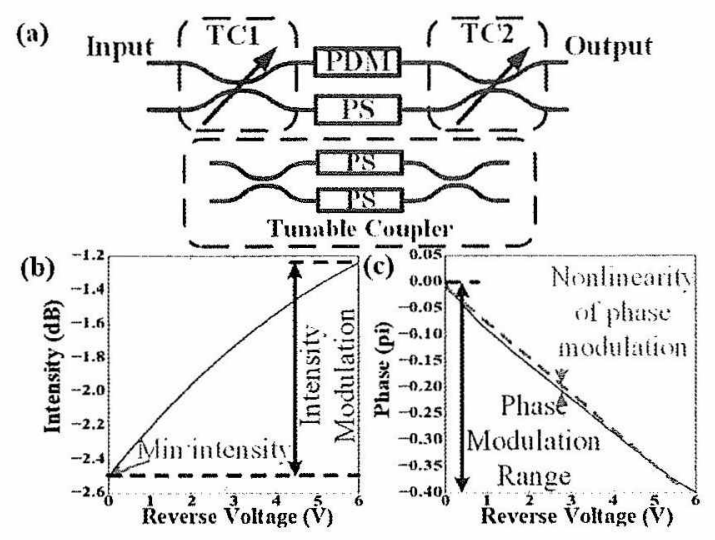

Fig. 1. (a) Schematic of the proposed contigurable modulator circuit. PS: (static) phase shifter; PDM: plasma dispersion modulator; TC: tunable coupler: (b), (c) Intensity and phase response of a stand-alone PN modulator [4].

\section{PRINCIPLE AND SIMULATION}

The schematic of the proposed configurable modulator is shown in Fig. 1(a). The circuit is an MZI built by two TCs, a plasma dispersion modulator (PDM) and a static phase shifter. The PDM in one arm is a simple PN junction phase modulator, while the phase shifter on the other arm tunes the static phase delay between the arms. The TCs can be implemented as simple balanced MZIs with their own static phase shifter [5]. All slow/static phase shifters can be realized by heaters.

The fast PN junction phase modulator changes the refractive index of the silicon waveguide and introduces a phase shift to the guided light wave, by removing or injecting free carriers in the waveguide core when reversed biased (depletion) or forward biased (injection). A typical intensity and phase response of a PN modulator is shown in Fig. 1(b) and (c)[4] . As shown, the bias voltage introduces both a phase modulation and an (unwanted) intensity modulation, and the response is not rigorously linear. However, when the modulator is embedded in a configurable MZI structure, the transfer function of the configurable modulator circuit can be described as [3]:

$H=\left[\sqrt{\left(1-\kappa_{1}\right)\left(1-\kappa_{2}\right)} \alpha(V) e^{-j \phi_{m}(V)}+\sqrt{\kappa_{1} \kappa_{2}} e^{-j\left(\phi_{s}-\pi\right)}\right]$

in which $\kappa_{1}$ and $\kappa_{2}$ are the splitting ratios of the TC1 and TC2, $\alpha(V)$ and $\phi_{m}(V)$ are the intensity and phase response of the 

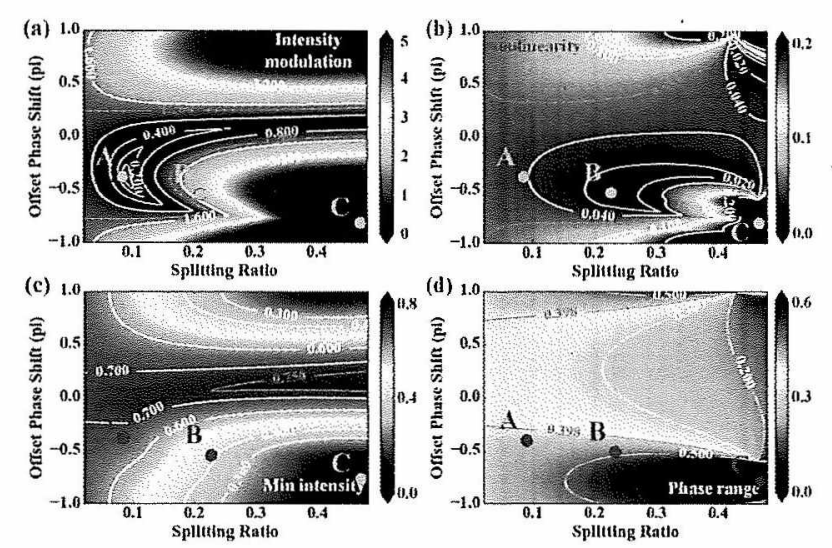

Fig. 2. Simulation of a $P N$ modulator with a $0-6 \mathrm{~V}$ drive voltage. We sweep the coupling $\kappa$ and the static phase shift $\phi_{s}$. (a) Spurious intensity modulation; (b) nonlinearity of the phase response; (c) minimal transmission (insertion loss); (d) phase modulation range. The green curve indicates the 'naked' PN modulator, without the circuit.

PDM, and $\phi_{s}$ is the phase shift induced by the static phase shifter. From Eq. 1, the total transmission of the configurable modulator is determined by the embedded modulator, the splitting ratios of the TCs and the phase shifts from the phase shifter, while the $\kappa_{1}$ and $\kappa_{2}$ are interchangeable. To assess the performance of the proposed modulator, we swept the offset phase shift $\phi_{s}$ and the coupling ratios, assuming $\kappa_{1}=\kappa_{2}=\kappa$ in the range $0 \leq \kappa \leq 0.5$, because it does not make sense to feed less light into the (lossy) modulator that in the other (lossless) arm. The simulation results are shown in Fig. 2, and more elaborate simulation exploration can be found in [3].

Normally, when $\kappa=0$, the light will entirely pass through the PDM and no interference occurs, and the circuit works as a traditional phase modulator. When $\kappa=0.5$, the light split evenly into the PDM and the other arm, and the circuit forms a classical MZI modulator (MZM), where $\phi_{s}$ tunes the bias point. Between these extremes of $\kappa=0$ and $k=0.5$, two operating points stand out. At point $A$, the intensity modulation is minimized, which results in an almost pure phase modulation. And at point $\mathrm{B}$, the phase response is the most linear. More metrics of the modulator can be explored in this parameter space and optimized operating points can be selected. Point $\mathrm{C}$ indicates the configuration with maximum intensity modulation and lowest phase modulation.

\section{EXPERIMENTAL RESULTS}

We fabricated this configurable modulator circuit in IMEC's iSiPP50G platform [6]; a photo is shown in Fig. 3(a). Fig. 3(bc) show the output power as function of drive voltage for three working states of the modulator. The voltage swing is set from $-10 \mathrm{~V}$ to $+1.5 \mathrm{~V}$. From $-10 \mathrm{~V}$ to $0.7 \mathrm{~V}$ (threshold voltage of PN junction) the PDM works as a depletion modulator, and with a higher voltage it works as an injection modulator. In Fig. 3(b), the orange line shows the circuit response as the "naked" PDM response, where the coupling ratio $\kappa$ of the TCs is set to 0 and all light goes through the PDM, corresponding
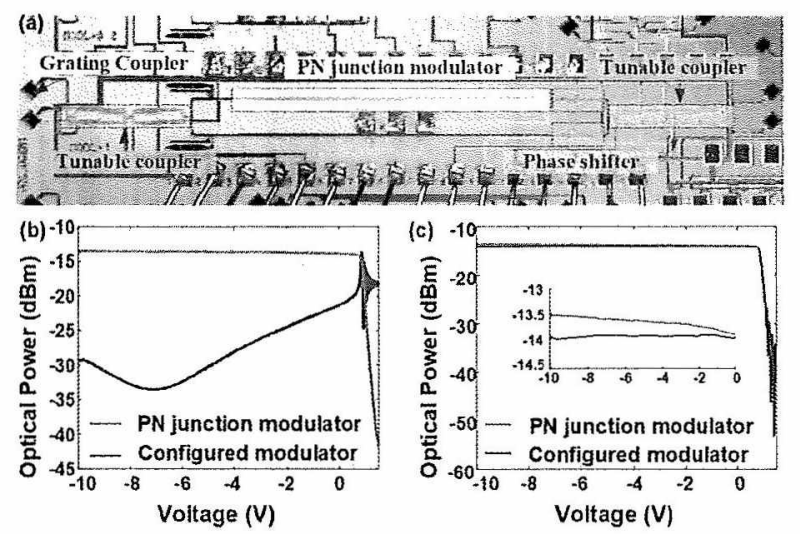

Fig. 3. (a) Photo of the fabricated circuit; (b) DC response of the modulator as phase modulator and as intensity modulator; (c) DC response of the modulator as pure phase modulator. The orange line: phase modulator: The blue line: intensity modulator. The green line: pure phase modulator:

to $x=0$ in Fig. 2. As shown, the insertion loss, and therefore the intensity modulation of the PDM increases gradually from $-10 \mathrm{~V}$ to $0.7 \mathrm{~V}$, and increases dramatically after $0.7 \mathrm{~V}$, which matches the threshold voltage of a PN junction. When the $\kappa$ of the TCs is set to near 0.5 , the full circuit works as an intensity modulator, and the DC response is the blue curve (Point $\mathrm{C}$ in Fig. 2) in Fig. 3(b). The bias point of the MZI can be tuned by the phase shifter. The extinction ratio of the MZI is more than 12dB. Fig. 3(c) shows another working state (in green), where the response is optimized for pure phase modulation (Point A in Fig. 2). As shown, the intensity change in the reverse biased region is reduced from $0.5 \mathrm{~dB}$ to around $0.06 \mathrm{~dB}$, but in the forward bias this setting will generate some interference, indicating that we are indeed sending light through both arms of the MZI to compensate the the spurious intensity modulation in reverse bias.

In conclusion, we proposed a configurable modulator circuit, and showed the DC responses when it is configured as an intensity modulator and a pure phase modulator.

\section{ACKNOWLEDGEMENTS}

This work was supported by the European Union through the ERC consolidator grant 725555 (PhotonicSWARM).

\section{REFERENCES}

11] D. Marpaung et al., "Tntegrated microwave photonics," Nature Photonics. vol. 13, no. 2, pp. 80-90, 22019.

[2] G. T. Reed et al. "Silicon optical modulators," Nature Photonics, vol. 4, no. 8 , pp. $518-526,2010$.

[3] H. Deng and W. Bogaerts, "Pure phase modulation based on a silicon plasma dispersion modulator," Optics Express, vol. 27, no. 19, p. 27191, 92019.

[4] H. Yu et al., "Performance tradeoff between lateral and interdigitated doping patterns for high speed carrier-depletion based silicon modulators," Optics Express, vol. 20 , no. 12 , p. $12926,62012$.

[5] K. Suzuki et al., "Ultra-high-extinction-ratio $2 \times 2$ silicon optical switch with variable splitter," Optics Express, vol. 23, no. 7, p. 9086, 2015.

[6] M. Pantouvaki et al., "Active Components for $50 \mathrm{~Gb} / \mathrm{s}$ NRZ-OOK Optical Interconnects in a Silicon Photonics Platform," Journal of Lightwave Tec/mology, vol. 35, no. 4, pp. 631-638, 2017. 


\section{WB1 - Configurable Phase/Amplitude Modulator Circuit bä Silicon Plasma Dispersion}

\begin{abstract}
A configurable modulator is demonstrated, implemented as a silicon Mach-Zehnder interferometer with a plasma dispersion modulator and tunable couplers. The configurable modulator can work as a phase modulator or intensity modulator with optimized modulation response.
\end{abstract}

TOPICS

Electronic-Photonic Integrated..

\author{
PRESENTED BY \\ Hong Deng \\ ( Beigium) - Gent University
}

\section{AUTHORS}

1. Hong Deng ( Belgium $)^{1}$

2. Wim Bogaerts (melgium) ${ }^{\prime}$

1. Gent University

MORE IN THIS SESSBON

Oral: WB

WB: Modulators II

8:30am - 9:45am

Wednesday, 8 December 2021

DESCRYPTION

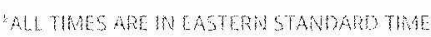

Vingsention 